\title{
RESEARCH
}

Open Access

\section{Paradata analyses to inform population- based survey capture of pregnancy outcomes: EN-INDEPTH study}

Vladimir Sergeevich Gordeev ${ }^{1,2^{*}}$, Joseph Akuze ${ }^{2,3,4}$, Angela Baschieri ${ }^{2}$, Sanne M. Thysen ${ }^{5,6,7}$, Francis Dzabeng ${ }^{8}$, M. Moinuddin Haider ${ }^{9}$, Melanie Smuk ${ }^{10}$, Michael Wild ${ }^{11}$, Michael M. Lokshin ${ }^{11}$, Temesgen Azemeraw Yitayew ${ }^{12}$, Solomon Mokonnen Abebe ${ }^{12}$, Davis Natukwatsa ${ }^{13}$, Collins Gyezaho ${ }^{13}$, Seeba Amenga-Etego ${ }^{8}$, Joy E. Lawn ${ }^{2 \dagger}$, Hannah Blencowe ${ }^{2 \dagger}$ and the Every Newborn-INDEPTH Study Collaborative Group

\begin{abstract}
Background: Paradata are (timestamped) records tracking the process of (electronic) data collection. We analysed paradata from a large household survey of questions capturing pregnancy outcomes to assess performance (timing and correction processes). We examined how paradata can be used to inform and improve questionnaire design and survey implementation in nationally representative household surveys, the major source for maternal and newborn health data worldwide.

Methods: The EN-INDEPTH cross-sectional population-based survey of women of reproductive age in five Health and Demographic Surveillance System sites (in Bangladesh, Guinea-Bissau, Ethiopia, Ghana, and Uganda) randomly compared two modules to capture pregnancy outcomes: full pregnancy history (FPH) and the standard DHS-7 full birth history $(\mathrm{FBH}+)$. We used paradata related to answers recorded on tablets using the Survey Solutions platform. We evaluated the difference in paradata entries between the two reproductive modules and assessed which question characteristics (type, nature, structure) affect answer correction rates, using regression analyses. We also proposed and tested a new classification of answer correction types.

(Continued on next page)
\end{abstract}

\footnotetext{
* Correspondence: v.gordeev@qmul.ac.uk; vladimir.gordeev@lshtm.ac.uk

† Joy E. Lawn and Hannah Blencowe are joint senior authors.

${ }^{1}$ Institute of Population Health Sciences, Queen Mary University of London, London, UK

${ }^{2}$ Maternal, Adolescent, Reproductive \& Child Health (MARCH) Centre, London School of Hygiene \& Tropical Medicine, London, UK

Full list of author information is available at the end of the article
}

(c) The Author(s). 2021 Open Access This article is licensed under a Creative Commons Attribution 4.0 International License, which permits use, sharing, adaptation, distribution and reproduction in any medium or format, as long as you give appropriate credit to the original author(s) and the source, provide a link to the Creative Commons licence, and indicate if changes were made. The images or other third party material in this article are included in the article's Creative Commons licence, unless indicated otherwise in a credit line to the material. If material is not included in the article's Creative Commons licence and your intended use is not permitted by statutory regulation or exceeds the permitted use, you will need to obtain permission directly from the copyright holder. To view a copy of this licence, visit http://creativecommons.org/licenses/by/4.0/. The Creative Commons Public Domain Dedication waiver (http://creativecommons.org/publicdomain/zero/1.0/) applies to the data made available in this article, unless otherwise stated in a credit line to the data. 
(Continued from previous page)

Results: We analysed 3.6 million timestamped entries from 65,768 interviews. 83.7\% of all interviews had at least one corrected answer to a question. Of 3.3 million analysed questions, 7.5\% had at least one correction. Among corrected questions, the median number of corrections was one, regardless of question characteristics. We classified answer corrections into eight types (no correction, impulsive, flat (simple), zigzag, flat zigzag, missing after correction, missing after flat (zigzag) correction, missing/incomplete). 84.6\% of all corrections were judged not to be problematic with a flat (simple) mistake correction. Question characteristics were important predictors of probability to make answer corrections, even after adjusting for respondent's characteristics and location, with interviewer clustering accounted as a fixed effect. Answer correction patterns and types were similar between $\mathrm{FPH}$ and $\mathrm{FBH}+$, as well as the overall response duration. Avoiding corrections has the potential to reduce interview duration and reproductive module completion by $0.4 \mathrm{~min}$.

Conclusions: The use of questionnaire paradata has the potential to improve measurement and the resultant quality of electronic data. Identifying sections or specific questions with multiple corrections sheds light on typically hidden challenges in the survey's content, process, and administration, allowing for earlier real-time intervention (e.g., questionnaire content revision or additional staff training). Given the size and complexity of paradata, additional time, data management, and programming skills are required to realise its potential.

Keywords: Survey, Paradata, Neonatal, Newborn, Answer correction type, Survey design

\section{Key findings}

\section{What is new?}

- What was known already: Paradata are widely used in the field of survey methodology in high-income countries to monitor on-going fieldwork progress and identify issues with specific questions but have been little-used to date in low- and middle-income countries and for maternal, newborn, and child health data collection or research. -What was done: We analysed paradata from the EN-INDEPTH survey administered to 65,768 women of reproductive age in five countries. We assessed which question characteristics used to capture pregnancy outcomes affected duration of section completion, data correction rates, or were associated with multiple corrections and whether these differed by two maternity history modules (full pregnancy history (FPH) and full birth history $(\mathrm{FBH}+))$.

\section{What was found?}

- Corrections to questions were common: affecting $83.7 \%$ of interviews, with a median of two questions corrected per interview and one correction per question when corrected (maximum of 28 corrections). $7.5 \%$ of the 3.3 million questions analysed had at least one correction.

- Simple one-time corrections most common: accounting for $84.6 \%$ of all corrections.

- In variation in corrections by maternity history module: number and type of corrections were similar between FPH and FBH+.

- In variation in corrections by question characteristics: number and type of corrections were affected by question characteristics. The proportion of corrected questions was 3.3\% higher for questions with notifications (9.8\%) than for questions without notifications (6.5\%).

- Duration of question completion: was not affected by question characteristics (type, content, structure) or history type. Avoiding corrections has the potential to reduce interview duration and reproductive module completion by $0.4 \mathrm{~min}$.

\section{What next in measurement and research?}

- Measurement improvement now: Paradata can be used to identify questions with multiple corrections, informing question editing or targeted training during and after survey completion. Encoding ranges and instant error notifications in the reproductive modules could reduce data missingness and prompt for timely data correction. Paradata analyses are skill- and time-consuming, but, if automatised, can be used for real-time data collection monitoring and data quality control.

\section{Key findings (Continued)}

- Research needed: Studies could examine interviewer productivity and possible fatigue related to the length of the interview, the number of corrections, and correction types. The real-time dashboard monitoring and reporting systems using paradata could be evaluated in terms of associations with data quality and usefulness for survey management. Qualitative interviews with both respondents and interviewers would help to identify and verify factors affecting correction frequency to inform better questionnaire design and training adjustment.

\section{Background}

High-quality routine health data on maternal, newborn, and child health $(\mathrm{MNCH})$ can be used to monitor, identify gaps, and take action to improve quality of care, optimise health system performance, and enable informed decision-making. Routine health management information systems vary in their completeness and quality across lowand middle-income countries (LMICs), and in many cases, are not able to provide the high-quality coverage data required for assessing and guiding health programmes $[1,2]$. Household surveys, notably Demographic and Health Surveys (DHS) and Multiple Indicator Cluster Surveys (MICS), remain the primary sources of data for the outcome and coverage indicators for children and women for most low- and middle-income countries. However, despite existing quality control mechanisms in the survey process, data quality, including missingness, age displacement, and heaping, remains a challenge [3]. Optimising survey data efficiency and quality requires more information regarding the survey process and performance [4].

The shift from paper-based to computer-assisted personal interviewing (CAPI)-based data collection (e.g., using tablets and smartphones) has enabled inclusions of inbuilt validation and consistency checks, as well as a real-time 
review of collected data [5]. In addition to the main survey dataset (which contains only the final respondent's answers), it is also possible to collect the survey's paradata (Fig. 1). Paradata contain information on the process of how data for each observation in the main survey dataset was collected and include detailed timestamped records of all survey actions including survey administration, interview process, as well as a detailed history of all the survey's data entry and correction [6, 7]. For example, paradata can show the order in which the questions were answered or corrected and reveal the content of deleted responses, which otherwise are not stored in the main survey dataset.

Even though paradata are widely used in the field of survey methodology in high-income countries [8], the use to date in LMICs and MNCH research has been limited. In household surveys, timestamped paradata can be used to monitor ongoing fieldwork progress and identify issues with specific questions or questionnaire sections based on the length of interviews or item response time [8]. Additional analyses can identify drivers behind item non-response and response time (e.g., survey locale; interviewer or respondent characteristics; survey's content-questions' type, nature, and structure) [9]. Paradata can also reveal determinants of data correction in relevant core survey questions during interviews as well as answer correction patterns (even though there is currently no agreed standardised terminology). Hence, paradata could lead to the overall improvements in data quality through targeted training [8] as well as improving questionnaire and survey design (structure and content) and survey implementation (process).

In this paper, we examine how paradata can be used to inform and improve questionnaire design and survey implementation in a large household survey collecting information on pregnancies and births using full pregnancy histories (FPH) and full birth histories with additional questions on pregnancy losses in the past 5 years $(\mathrm{FBH}+)$. This paper is one of a series of papers from the Every Newborn International Network for the Demographic Evaluation of Populations and Their Health (EN-INDEPTH) study in five Health and Demographic Surveillance System (HDSS) sites in sub-Saharan Africa and Asia.

This paper has three objectives:

1. To assess the differences in paradata timestamped entries between two reproductive modules (FPH and $\mathrm{FBH}+$ );

2. To determine whether question characteristics (type, nature, structure) affect the duration of section completion and answer correction rates;

3. To propose and test classification of answer correction types and determine whether they differ by two reproductive modules.

\section{Methods}

Overall EN-INDEPTH study design and data sources The EN-INDEPTH study aimed to compare two approaches of collecting maternity history ( $\mathrm{FPH}$ and $\mathrm{FBH}+$ ) to examine whether the two methods yield different estimates of stillbirth rates and neonatal mortality rates and to determine whether there is a difference in completion time for these two approaches. The study protocol and main findings can be found elsewhere [10, 11]. Briefly, the EN-INDEPTH survey reached 69,176 women of reproductive age in five HDSS sites (Bandim in Guinea-Bissau, Dabat in Ethiopia, IgangaMayuge in Uganda, Matlab in Bangladesh, and Kintampo in Ghana). Participants of the EN-INDEPTH study were randomly assigned (1:1) to be interviewed using a questionnaire containing either an $\mathrm{FPH}$ or an $\mathrm{FBH}+$ module (section 2 in Fig. 2). The EN-INDEPTH study used the World Bank's Survey Solutions CAPI/CAWI (computer-assisted web interviewing) data collection and management platform (hereafter Survey Solutions) [12] to collect face-to-face responses to the questionnaire (Additional file 1). The choice of the

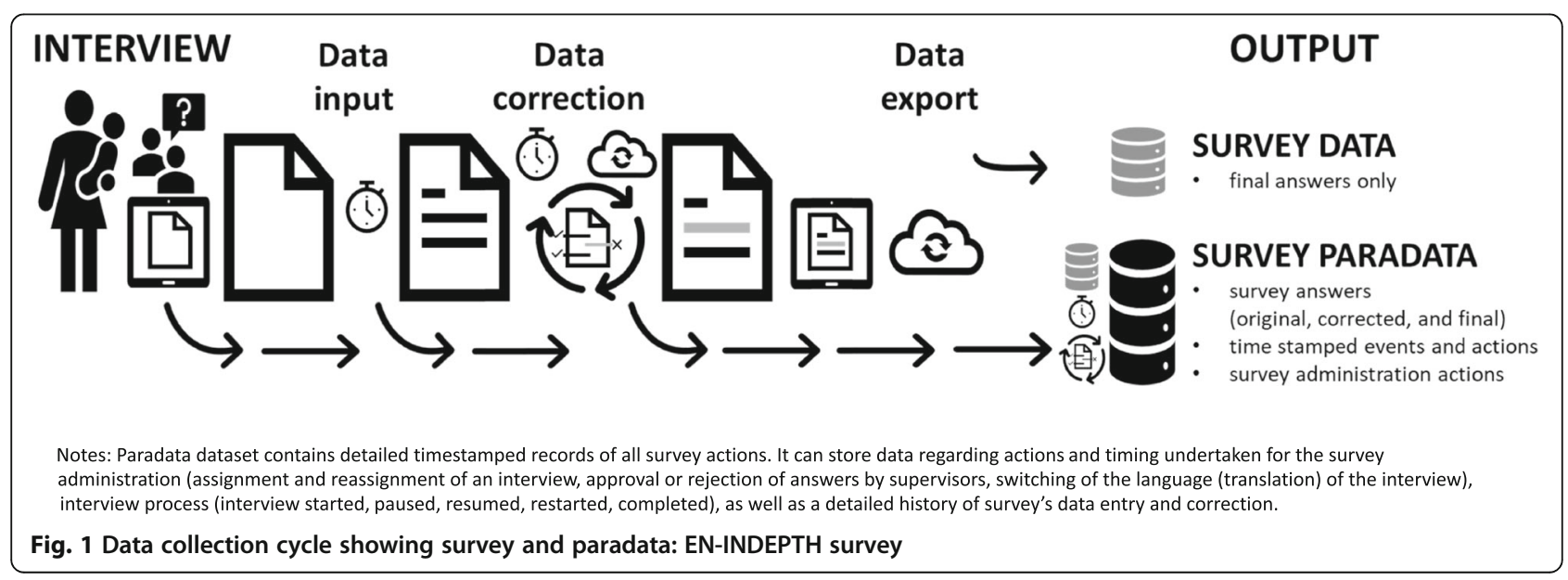




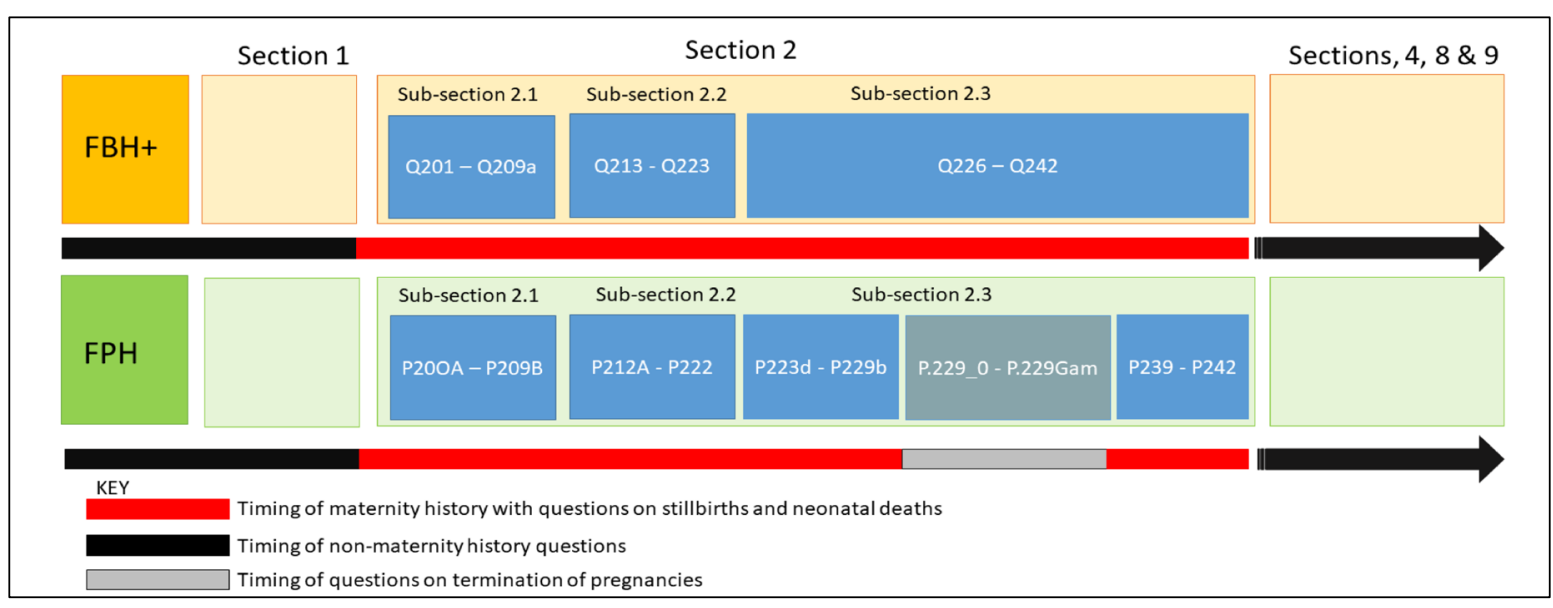

Notes: FPH - full pregnancy history module. FBH+ - full birth history module with additional questions on pregnancy losses. Section 1 - Consent form and background of an interview; Section 2 - Reproduction module; Section 4 - Pregnancy and postnatal care; Section 8 - Fertility preferences; Section 9 - Household characteristics.

Fig. 2 Module structure for questions in the two arms of the EN-INDEPTH survey

software and an overview of the data collection process and procedures are detailed elsewhere [13].

The analyses in this paper focus on the reproduction section (section 2) of the questionnaire only (Fig. 2). Section 2 contains three subsections. In subsection 2.1, women were asked to state their total lifetime number of liveborn children (FPH and $\mathrm{FBH}+$ ) and a total number of pregnancy losses (FPH only). In subsection 2.2, women were asked for details about their lifetime pregnancies $(\mathrm{FPH})$ or lifetime livebirths $(\mathrm{FBH}+)$ and answer a subset of questions for each instance. In subsection 2.3, women were asked in the $\mathrm{FBH}+$ group about pregnancy losses in the past 5 years, while the FPH module contained an additional set of questions on termination of pregnancy (TOP), which we also included in the analyses (Additional file 1).

\section{Data processing}

Paradata were exported in a tab-delimited format from the Survey Solutions platform [12], with each line corresponding to one recorded event (example in Additional file 2). Data from all sites were fully anonymised and required subsets of data extracted and merged using the $R$ software [14]. We included only timestamped entries related to answers and corrections from section 2. We excluded entries that related to assigned but never conducted interviews and duplicate entries that resulted from updating questionnaire and software. All subsequent analyses were performed using Stata 16.1 [15].

\section{Methods by objective}

Objective 1: To assess differences in paradata timestamped entries between two reproductive modules (FPH and $\mathrm{FBH}+$ )

Time taken to complete a question was estimated based on the difference between the timestamps of the previously answered question and the current one (based on answered questions order) and separate per observation (in case of parity $>1$ ). For questions with corrections, the timestamp of the final answer was taken as the question's timestamp. To exclude implausible values, but allow for multiple corrections during the module completion and/or switching between questionnaire sections during the interview [16], we restricted analyses of time taken to complete maternity history section of the survey to interviews lasting $0-180 \mathrm{~min}$.

We categorised all questions by type, nature, and structure. The question types included single-select (e.g., "Was that baby a twin?'); multi-select (e.g., 'Who assisted with the delivery of this baby?'); numerical computational (e.g., 'How many children do you have?'); date-related (e.g., 'What was the date of birth for this baby?); and free-text input (e.g., 'What is the name of your baby?). There were three categories based on questions' nature: two groups of potentially sensitive questions (death-related-relating to death and/or pregnancy loss, and TOP-related questions) and regular (non-sensitive) questions. Lastly, considering question structure, there were questions with built-in error notifications (e.g., displaying "value outside the range, please correct"), warnings appearing in capital red letters, or any other prompts for correction (e.g., when answers for age based on birth and age at last birthday did not match) and those without such notifications. Differences between $\mathrm{FPH}$ and $\mathrm{FBH}+$ were evaluated using descriptive statistics and independent sample $t$ test. Statistical significance level was defined at the $5 \%$ level.

\section{Objective 2: To determine whether question characteristics affect the duration of section completion and answer correction rates}

Differences in the duration of response time and proportion of corrections by question characteristics 
(type, nature, structure) were evaluated using descriptive statistics. A two-part model was used to analyse which question characteristics are associated with the likelihood of question correction (generalised linear model (GLM) with a binomial distribution and logit link) and the number of corrections (GLM with gamma distribution and log link function). Explanatory variables included question characteristics. Models were adjusted for respondent's characteristics and location, with interviewer clustering accounted as a fixed effect. Statistical significance level was defined at the $5 \%$ level.

\section{Objective 3: To propose and test classification of answer correction patterns and determine whether data correction patterns differ by two reproductive modules}

In paradata, the process of data collection where all answers are entered and corrected is recorded as an ordered list of answers (sequence). To understand this process of data entry and correction better, we ordered all interviews based on the total number of questions asked during the interview and the number of answers (length of a sequence). We distinguished between original answers and corrections and visually inspected the resulting sequence index plot [17].

Whenever an answer to the same question has multiple corrections, these corrections can form a distinct pattern. For example, corrections can be single or multiple; the value of the original answer and the last correction may or may not match; correction entries may have identical or different values and may lead to missing data. As currently there is no classification of answer correction types, we developed and tested one using our survey data. We then used descriptive statistics to examine whether answer correction patterns vary by question characteristics and two reproductive modules.

Results are reported in accordance with STROBE Statement checklists for cross-sectional studies [18] (Additional file 3).

\section{Results}

\section{Objective 1: To assess differences in paradata} timestamped entries between two reproductive modules (FPH and $\mathrm{FBH}+$ )

\section{Number of timestamped entries}

We analysed 3.6 million timestamped entries corresponding to 3.3 million answered questions and their correction for 65,768 interviews, of which $52.1 \%$ related to FPH module (32,744 interviews), which by design contained more questions than the $\mathrm{FBH}+$ reproductive module (33,024 interviews) (Table 1 and Additional file 4). Among all entries, $18.5 \%$ related to the pregnancy or birth history (sub-section 2.1), 66.2\% to the roster (sub-section 2.2), and $15.3 \%$ to reproduction subsections of $\mathrm{FPH}$ and $\mathrm{FBH}+$ reproductive modules (sub-section 2.3) (Fig. 2). The median number of timestamped answers per interview was 48 (52 and 45 for $\mathrm{FPH}$ and $\mathrm{FBH}+$, respectively).

\section{Type, nature, and structure of questions}

$\mathrm{FPH}$ and $\mathrm{FBH}+$ modules contain 98 and 66 possible uniquely formulated question/answer fields, respectively (Additional files 1 and 4). FPH reproductive module contains 52 single-select questions, 26 numerical computational, one date-related, and 17 free-text and two multi-select types of questions. FBH+ module contains 35 single-select questions, 28 numerical computational, one date-related, and two free-text and no multi-select types of questions. FPH has 18 questions related to death/pregnancy loss and 39 questions related to TOP (including country-specific questions). FBH has 27 questions related to pregnancy loss/death. The rest of the questions are regular (non-sensitive) by nature. A quarter of questions in FPH and about roughly a third in $\mathrm{FBH}+$ have built-in error notifications.

Most of the timestamped entries related to single-select questions (66.6\%), followed by numerical computational (32.1\%), date-related (1.2\%), and less than $0.03 \%$ being free-text and multi-select types of questions (Additional file 4). The proportion of timestamped entries per question type between modules was very similar. In terms of the questions' nature, most of the timestamped entries were for regular questions (87.2\%) and not related to the two groups of potentially sensitive questions (death and/ or pregnancy loss, and TOP-related questions). In terms of structure, about a third of timestamped entries were for questions that had built-in error notifications, warnings, or other prompts for a correction.

\section{The average duration of section and question completion}

The median number of questions answered per one interview was 44: 49 for $\mathrm{FPH}$ and 41 for $\mathrm{FBH}+$, as FPH contained an additional set of TOP-related questions absent in $\mathrm{FBH}+$. The median duration of section 2 completion was 7.3 min (Table 1, Fig. 3). The average time taken to complete the reproduction module was $1.1 \mathrm{~min}$ longer for the FPH (mean $=11.4 \mathrm{~min}$ ) than the $\mathrm{FBH}+(10.3 \mathrm{~min})$. The median response time per question was around $0.1 \mathrm{~min}$ overall and for both modules.

\section{Corrections per interview and questions}

Overall, $83.7 \%$ of all interviews had at least one corrected answer to a question, slightly higher for FPH module than $\mathrm{FBH}+(84.6 \%$ and $82.8 \%$, respectively $)$ (Table 1). The median number of corrected questions per interview was two, and a median number of corrections was three. The median time spent on corrections per one interview was 0.3 min (the mean 
Table 1 Interview process details: number of timestamped entries, response time and corrections

\begin{tabular}{|c|c|c|c|c|c|c|c|c|c|c|}
\hline \multirow[t]{2}{*}{ Indicator } & \multicolumn{3}{|l|}{ Overall } & \multicolumn{3}{|l|}{$\mathrm{FPH}$} & \multicolumn{3}{|l|}{$\mathrm{FBH}+$} & \multirow[t]{2}{*}{$P$ value } \\
\hline & Mean (SD) & Median & Range & & Median & Range & Mean (SD) & Median & Range & \\
\hline $\begin{array}{l}N \text { timestamped entries per } \\
\text { interview }^{\mathrm{a}}\end{array}$ & $55.1(33.4)$ & 48 & $1-335$ & $57.7(34.9)$ & 52 & $1-335$ & $52.5(31.5)$ & 45 & $6-289$ & $<0.001$ \\
\hline $\begin{array}{l}N \text { questions answered per } 1 \\
\text { interview }\end{array}$ & 50.7 (29.6) & 44 & $1-223$ & $53.2(31.1)$ & 49 & $1-223$ & $48.4(27.8)$ & 41 & 6-194 & $<0.001$ \\
\hline $\begin{array}{l}\text { Response time per } 1 \text { interview }{ }^{b} \text {, } \\
\text { min }\end{array}$ & $10.8(14.3)$ & 7.3 & $0.06-179.9$ & $11.4(14.8)$ & 7.8 & $0.06-179.9$ & $10.3(13.3)$ & 6.9 & $0.23-179.8$ & $<0.001$ \\
\hline $\begin{array}{l}\text { Response time per } 1 \text { question }{ }^{\mathrm{b}} \text {, } \\
\text { min }\end{array}$ & $0.4(3.5)$ & 0.08 & $0-179.9$ & 0.4 (3.6) & 0.08 & $0-179.9$ & $0.4(3.6)$ & 0.07 & $0-179.9$ & \\
\hline $\begin{array}{l}N \text { corrected questions per } 1 \\
\text { interview }\end{array}$ & $3.8(4.8)$ & 2 & $0-112$ & $3.9(4.9)$ & 3 & $0-112$ & $3.6(4.8)$ & 2 & $0-110$ & $<0.001$ \\
\hline $\begin{array}{l}N \text { corrected question per } 10 \\
\text { questions }\end{array}$ & $0.8(0.9)$ & 0.6 & $0-29.5$ & $0.8(0.9)$ & 0.6 & $0-29.5$ & $0.8(0.9)$ & 0.62 & $0-20.4$ & $<0.01$ \\
\hline$N$ corrections per 1 interview & $4.4(6.2)$ & 3 & $0-227$ & $4.6(6.3)$ & 3 & $0-227$ & $4.2(6.1)$ & 2 & $0-149$ & $<0.01$ \\
\hline $\begin{array}{l}\text { Time spent on correction per } 1 \\
\text { interview, min }\end{array}$ & $1.9(10.2)$ & 0.3 & $0-179.6$ & $2.0(10.2)$ & 0.3 & $0-174.5$ & $1.9(10.2)$ & 0.2 & $0-179.6$ & $<0.01$ \\
\hline $\begin{array}{l}\text { Time spent on correction per } 1 \\
\text { question, min }\end{array}$ & $0.6(5.6)$ & 0.08 & $0-179.9$ & $0.6(5.5)$ & 0.08 & $0-179.8$ & $0.6(5.6)$ & 0.08 & $0-179.9$ & \\
\hline $\begin{array}{l}\text { Response time per } 1 \text { interview, } \\
\text { if all corrections avoided, min }\end{array}$ & $9.4(10.1)$ & 6.9 & $0.06-179.5$ & $9.9(10.6)$ & 7.4 & $0.06-179.5$ & $8.8(9.4)$ & 6.5 & $0.2-179.4$ & $<0.001$ \\
\hline $\begin{array}{l}\text { Response time per } 1 \text { question, } \\
\text { if corrections avoided, min }\end{array}$ & $1.7(4.5)$ & 0.8 & $0.5-177.1$ & $1.7(4.5)$ & 0.8 & $0.5-177.1$ & $1.7(4.5)$ & 0.9 & $0.5-176.1$ & \\
\hline$N$ interviews, $n(\%)$ & \multicolumn{3}{|c|}{$65,768(100.0)$} & \multicolumn{3}{|c|}{$32,744(49.8)$} & \multicolumn{3}{|l|}{$33,024(50.2)$} & \\
\hline $\begin{array}{l}N \text { interviews with at least } 1 \\
\text { correction, } \mathrm{n}(\%)\end{array}$ & \multicolumn{3}{|c|}{$55,066 / 65,768(83.7)$} & \multicolumn{3}{|c|}{$27,721 / 32,744$ (84.6) } & \multicolumn{3}{|c|}{$27,345 / 33,024(82.8)$} & \\
\hline
\end{tabular}

Total percentages may not add up or exceed one hundred due to rounding up

$F P H$ full pregnancy history module, $F B H+$ full birth history module with additional questions on pregnancy losses

${ }^{\$} P$ values for independent sample $t$ test that compared means for two groups

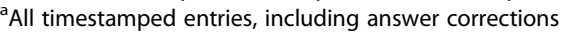

${ }^{b}$ All answers, accounting for correction time

time spent on correction was 0.1 min longer for $\mathrm{FPH}$ than $\mathrm{FBH}+$ ), and the median time to correct one question was $0.08 \mathrm{~min}$. Without corrections, the median response time per interview would be lower by $5.5 \%$ or $0.4 \mathrm{~min}$.

\section{Objective 2: To determine whether question characteristics (type, nature, structure) affect the duration of section completion and answer correction rates \\ Duration of question completion}

The median response time per question type was longest for free-text and multi-select questions $(0.6 \mathrm{~min})$, followed by date-related $(0.2 \mathrm{~min})$, numerical computational $(0.1 \mathrm{~min})$, and single-select questions $(0.05 \mathrm{~min})$ (Fig. 4). The median response time only slightly varied per question's nature, with the longest median response time for TOP-related questions $(0.1 \mathrm{~min})$. Questions with built-in error notifications had a median response time of $0.1 \mathrm{~min}$ compared with 0.07 min for questions with no built-in error notifications.

\section{Proportion of corrections}

Of all asked and answered questions, 7.5\% had at least one or more corrections (Fig. 4, Additional file 5). Among all questions, the most frequently (by absolute number) corrected questions were single-select and numerical computational types of questions, regular questions, and questions without built-in notifications. However, the highest proportion of corrections within the question type group was multi-select questions (44.8\%), followed by free-text (16.2\%), numerical computational (9.7\%), singleselect (6.6\%), and date-related (4\%) questions. The proportion of corrections was similar based on the question's nature (around 6-8\%). The proportion of corrected questions was $3.3 \%$ higher for questions with notifications (9.8\%) than for questions without notifications (6.5\%).

Among corrected questions, the median number of corrections was one, regardless of question characteristics. The maximum number of corrections was the highest for single-select $(n=28)$ and numerical computational $(n=23)$ types of questions. In terms of questions' nature, the maximum number of corrections was among regular questions 


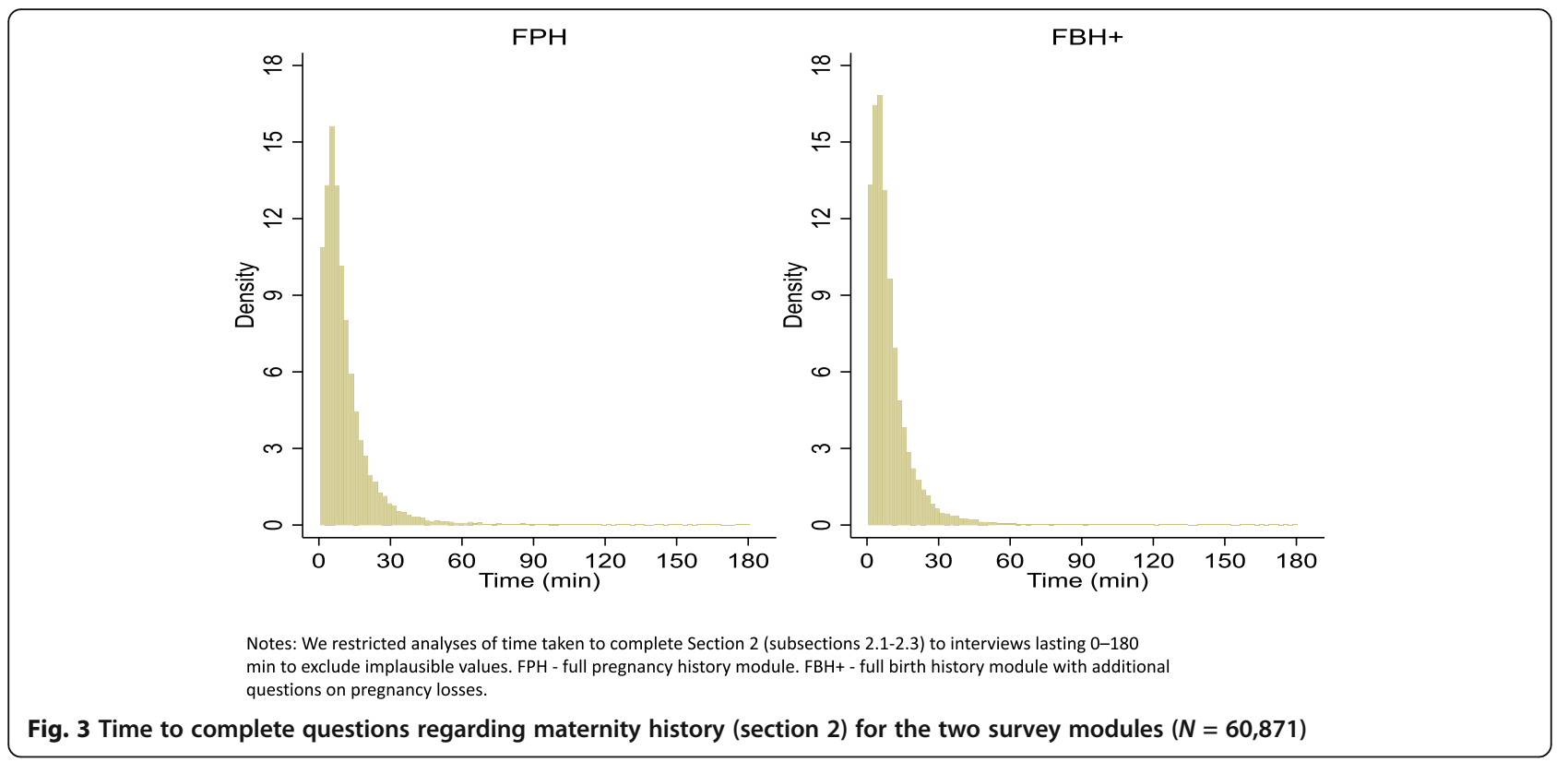

( $n=28)$, followed by death-related questions $(n=23)$ and TOP-related questions $(n=19)$. Regarding the question structure, the maximum number of corrections was highest for questions with no built-in notifications $(n=28)$.

Based on the results of the regression analyses (Table 2, model 1), date question type (reference-single) and death- related questions (reference-regular) decreased the probability of making corrections. All other question characteristics increased the probability of making answer corrections when compared to their reference groups. Question characteristics (numeric, date, multi-select, death- and TOP-related) were positively associated

\begin{tabular}{|c|c|c|c|c|c|c|c|c|c|c|}
\hline \multirow{3}{*}{$\begin{array}{l}\text { Question } \\
\text { characteristics }\end{array}$} & \multicolumn{7}{|c|}{ All questions } & \multicolumn{3}{|c|}{ Per corrected question } \\
\hline & \multicolumn{2}{|c|}{$\mathrm{N}$ questions asked } & \multicolumn{2}{|c|}{$\begin{array}{l}\text { N questions with } \\
\text { corrections }\end{array}$} & \multicolumn{3}{|c|}{ Response time per 1 question, $\min$} & \multicolumn{2}{|c|}{$\mathbf{N}$ corrections } & \multirow{2}{*}{$\begin{array}{c}\begin{array}{c}\text { Max N } \\
\text { corrections }\end{array} \\
n\end{array}$} \\
\hline & $\mathrm{n}$ & $\%$ & $\mathrm{n}$ & $\%$ & Mean (SD) & Median & Range & Mean (SD) & Median & \\
\hline Overall & $3,340,749$ & & 251,168 & 7.5 & $0.37(3.60)$ & 0.08 & $0-179.9$ & $1.14(0.51)$ & 1 & 28 \\
\hline \multicolumn{11}{|l|}{ Type } \\
\hline Single-select & $2,248,565$ & 67.3 & 147,713 & 6.6 & $0.33(3.35)$ & 0.05 & $0-179.9$ & $1.14(0.49)$ & 1 & 28 \\
\hline Multi-select & 192 & 0.01 & 86 & $44.8^{*}$ & $1.99(4.95)$ & 0.58 & $0.07-48.0$ & $2.13(2.33)$ & 1 & 19 \\
\hline $\begin{array}{r}\text { Numerical } \\
\text { computational }\end{array}$ & $1,048,996$ & 31.4 & 101,611 & 9.7 & $0.46(4.03)$ & 0.10 & $0-179.9$ & $1.15(0.52)$ & 1 & 23 \\
\hline Date-related & 42,582 & 1.3 & 1,691 & 4.0 & $0.43(2.70)$ & 0.20 & $0-176.1$ & $1.19(0.64)$ & 1 & 8 \\
\hline Free-text & 414 & 0.01 & 67 & $16.2^{*}$ & $2.14(9.25)$ & 0.58 & $0-139.3$ & $1.21(0.59)$ & 1 & 5 \\
\hline \multicolumn{11}{|l|}{ Nature } \\
\hline Regular & $2,913,144$ & 87.2 & 221,948 & 7.6 & $0.37(3.58)$ & 0.08 & $0-179.9$ & $1.13(0.48)$ & 1 & 28 \\
\hline Death-related & 346,112 & 10.4 & 22,673 & 6.6 & $0.37(3.66)$ & 0.07 & $0-179.9$ & $1.22(0.68)$ & 1 & 23 \\
\hline TOP-related & 81,493 & 2.4 & 6,547 & 8.0 & $0.39(3.11)$ & 0.13 & $0-164.2$ & $1.19(0.62)$ & 1 & 19 \\
\hline \multicolumn{11}{|l|}{ Structure } \\
\hline $\begin{array}{r}\text { Built-in error } \\
\text { notification }\end{array}$ & $1,012,871$ & 30.3 & 99,538 & 9.8 & $0.45(3.95)$ & 0.10 & $0-179.9$ & $1.15(0.52)$ & 1 & 23 \\
\hline $\begin{array}{r}\text { No built-in error } \\
\text { notification }\end{array}$ & $2,327,878$ & 69.7 & 151,630 & 6.5 & $0.34(3.40)$ & 0.07 & 0-179.9 & $1.14(0.50)$ & 1 & 28 \\
\hline
\end{tabular}

Notes: Total percentages may not add up or exceed one hundred due to rounding up. Subjective correction proportion colour-coding: green - 0-5\% - excellent, lime - 6-10\% very good, yellow - $11-15 \%$ good, orange - $16-20 \%$ moderate, and red - > $20 \%$ - poor. ${ }^{*}$ - Results for guidance only and should be treated with caution, given the design, and purpose of the question.

Fig. 4 Question corrections by question type, nature and structure 
Table 2 Question characteristics associated with answer correction probabilities and frequencies

\begin{tabular}{|c|c|c|c|c|c|c|c|c|c|c|c|c|}
\hline \multirow{3}{*}{$\begin{array}{l}\text { Characteristics } \\
\text { Variables }\end{array}$} & \multicolumn{6}{|c|}{ Model (1) } & \multicolumn{6}{|c|}{ Model (2) } \\
\hline & \multicolumn{3}{|c|}{$\begin{array}{l}\text { Part } 1 \text { correction } \\
\text { (yes/no) }\end{array}$} & \multicolumn{3}{|c|}{$\begin{array}{l}\text { Part } 2 \text { number } \\
\text { of corrections }\end{array}$} & \multicolumn{3}{|c|}{$\begin{array}{l}\text { Part } 1 \text { correction } \\
\text { (yes/no) }\end{array}$} & \multicolumn{3}{|c|}{$\begin{array}{l}\text { Part } 2 \text { number } \\
\text { of corrections }\end{array}$} \\
\hline & Coef & $\begin{array}{l}\text { Robust } \\
\text { Std. Err. }\end{array}$ & $\begin{array}{l}p \\
\text { value }\end{array}$ & Coef & $\begin{array}{l}\text { Robust } \\
\text { Std. Err. }\end{array}$ & $\begin{array}{l}p \\
\text { value }\end{array}$ & Coef & $\begin{array}{l}\text { Robust Std. } \\
\text { Err. }\end{array}$ & $\begin{array}{l}p \\
\text { value }\end{array}$ & Coef & $\begin{array}{l}\text { Robust } \\
\text { Std. Err. }\end{array}$ & $\begin{array}{l}p \\
\text { value }\end{array}$ \\
\hline \multicolumn{13}{|l|}{ Type, Ref (single) } \\
\hline $\begin{array}{l}\text { Numerical } \\
\text { computational }\end{array}$ & 0.056 & 0.012 & $<0.001$ & 0.050 & 0.007 & $<0.001$ & -0.138 & 0.014 & $<0.001$ & 0.026 & 0.008 & 0.001 \\
\hline Date-related & -0.531 & 0.025 & $<0.001$ & 0.055 & 0.013 & $<0.001$ & -0.411 & 0.028 & $<0.001$ & 0.072 & 0.015 & $<0.001$ \\
\hline Free-text & 0.850 & 0.146 & $<0.001$ & 0.031 & 0.061 & 0.611 & 0.864 & 0.164 & $<0.001$ & 0.041 & 0.075 & 0.581 \\
\hline Multi-select & 2.248 & 0.145 & $<0.001$ & 0.590 & 0.118 & $<0.001$ & 2.337 & 0.158 & $<0.001$ & 0.598 & 0.123 & $<0.001$ \\
\hline \multicolumn{13}{|l|}{ Nature, Ref (regular) } \\
\hline Death-related & -0.166 & 0.009 & $<0.001$ & 0.079 & 0.004 & $<0.001$ & -0.187 & 0.011 & $<0.001$ & 0.078 & 0.005 & $<0.001$ \\
\hline TOP-related & 0.205 & 0.015 & $<0.001$ & 0.040 & 0.007 & $<0.001$ & 0.316 & 0.017 & $<0.001$ & 0.055 & 0.008 & $<0.001$ \\
\hline \multicolumn{13}{|c|}{ Structure, Ref (no notification) } \\
\hline Yes, with notification & 0.400 & 0.012 & $<0.001$ & -0.041 & 0.007 & $<0.001$ & 0.486 & 0.014 & $<0.001$ & -0.026 & 0.008 & 0.001 \\
\hline \multicolumn{13}{|l|}{ Module, Ref (FPH) } \\
\hline $\mathrm{FBH}+$ & 0.015 & 0.009 & 0.101 & -0.003 & 0.003 & 0.248 & 0.012 & 0.009 & 0.169 & -0.003 & 0.003 & 0.333 \\
\hline Constant & -2.661 & 0.007 & $<0.001$ & 0.125 & 0.002 & $<0.001$ & -2.818 & 0.056 & $<0.001$ & 0.1465 & 0.016 & $<0.001$ \\
\hline Observations & \multicolumn{3}{|l|}{$3,340,189$} & \multicolumn{3}{|l|}{250,608} & \multicolumn{3}{|c|}{$2,247,142$} & \multicolumn{3}{|l|}{152582} \\
\hline R-squared & \multicolumn{3}{|l|}{0.004} & \multicolumn{3}{|l|}{0.004} & \multicolumn{3}{|l|}{0.014} & \multicolumn{3}{|l|}{0.014} \\
\hline Root MSE & \multicolumn{3}{|l|}{0.263} & \multicolumn{3}{|l|}{0.511} & \multicolumn{3}{|l|}{0.250} & \multicolumn{3}{|l|}{0.494} \\
\hline
\end{tabular}

Model (1) unadjusted, model (2) adjusted for respondent's characteristics (age, education, parity, wealth quintile) and location, with interviewer clustering accounted as a fixed effect. Both models accounted for clustering of individual responses within individual women (interview)

$\mathrm{FPH}$ full pregnancy history module, $\mathrm{FBH}+$ full birth history module with additional questions on pregnancy losses

with the number of corrections. Questions with notifications were negatively associated with the number of corrections. There was not enough evidence of an association between belonging to either of the two reproductive modules with either the probability of making a correction or the number of corrections.

After adjusting for respondent's characteristics and location, with interviewer clustering accounted as a fixed effect (Table 2, model 2), all question characteristics remained significantly associated with the probability of making answer corrections when compared to their reference groups; however, the numeric type changed the direction of the association. Numeric, date, multi-select, and death- and TOP-related questions continued to be positively associated with the number of corrections, while the questions with notifications remained negatively associated with the number of corrections.

\section{Objective 3: To propose and test classification of answer correction types and determine whether they differ by two reproductive modules Correction patterns}

In line with our findings for objectives 1 and 2 , the visual inspection of the sequence index plot (Fig. 5) showed that most of the interviews had corrections to answers. Only a smaller number of shorter interviews seemed to have no or a limited number of corrections. As the number of asked questions during the interview increased, so did the number of answer corrections. Based on the number of corrections per question, we identified 23 correction patterns, ranging from one to 28 corrections (Additional file 6). Most questions had single correction (89.0\%), followed by multiple corrections (two and three corrections, $8.8 \%$ and $1.6 \%$, respectively). The remaining $0.6 \%$ of questions had four and more corrections per question. We also observed that among these correction patterns, the original answer (first entry) sometimes matched the final answer correction (last entry), while for others, it did not. Some patterns consisted of either repetitive sequences of identical entries or a combination of different entries.

\section{Correction types}

By combining characteristics of answer correction process (single vs multiple corrections; first and last answer match vs do not match) and correction pattern (different vs identical entries), we developed a classification of answer correction types. We distinguished between eight possible answer correction types after accounting for possibly missing answers after corrections (Table 3). Building on the 


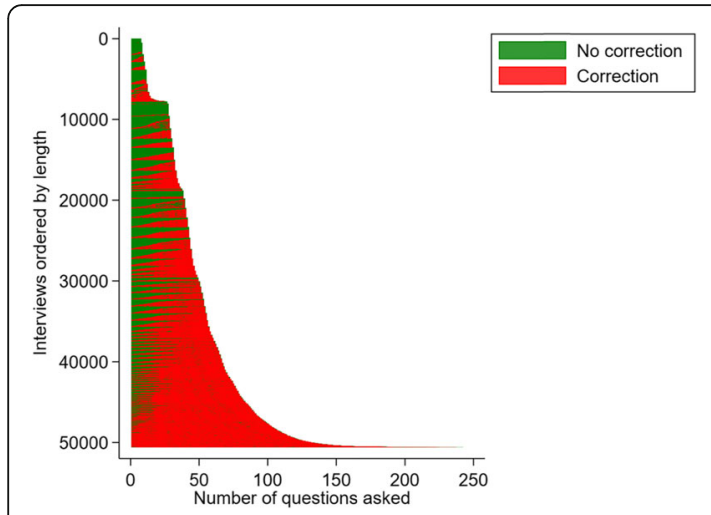

Notes: All interviews are presented as a horizontal sequence line, consisting of data entries with and without data correction. Correction group includes both original corrected questions and corrections. Interviews are sorted by the number of questions asked in Section 2 and the number of answers (sequence length). The red colour (representing corrected questions and corrections) might visually overlay and mask questions without corrections (presented by green colour) due to the resolution and a high number of interviews.

Fig. 5 Data collection and correction as a sequence index plot by the length of interview

original terminology used by Elliot (1934) for cycles and pattern market behaviours and using elements of the original terminology for corrective waves (i.e., flat, zigzag) [19], we named correction types as no correction, impulsive, flat (simple), zigzag, flat zigzag correction, missing after correction, missing after a flat (or zigzag) correction, missing, or incomplete. Additionally, we distinguished between non-problematic (no correction, repetitive entry of identical values, or accidental mistake) and problematic (multiple corrections, first and last entry do not match, or missing after correction) correction types.

We tested our proposed classification using our survey data. Out of eight proposed answer correction types (Table 3 ), we observed only five, including no correction type (Table 4). Among answers with corrections, a flat (or simple) correction was the most frequent answer correction type (84.6\%), followed by zigzag and flat zigzag corrections (5.3-5.5\%), and the impulsive correction type (4.6\%). As we did not treat the 'Do not know' as a missing answer and had no observations with truly missing answers, we did not observe the other four proposed answer correction types.

The flat correction type was the most frequently observed correction type (84.1\%), irrespective of question type, nature, or structure (Table 4). A zigzag correction was the second most frequent type of correction for single-select questions (6.9\%), while impulsive and flat zigzag correction types were the second most frequently observed correction types for daterelated (12.6\%) and numeric computational (33.7\%) question types, respectively. Accounting for the question's nature, a zigzag correction was the second most frequently observed correction type for death-related questions and TOP-related questions. For regular questions, the observed proportions were similar. Among questions with built-in notifications, the flat zigzag correction was the second most frequent type, while for questions with no built-in notifications, it was the zigzag correction.

\section{Discussion}

This is the first study to examine the feasibility and usefulness of paradata to enhance household survey capture of pregnancy outcomes to inform the content, timing, process, and administration of questions. We delve further into our earlier findings on the lack of statistically significant differences in response times for $\mathrm{FPH}$ or $\mathrm{FBH}+$ modules' completion [11]. Around $84 \%$ of interviews had at least one correction to questions; however, most of them were simple one-time corrections. We identified four out of eight proposed answer correction types (impulsive, flat (simple) correction, zigzag correction, and flat zigzag correction) and found that question characteristics (type, content, structure) could affect the probability of making answer correction, be associated with the number of corrections, and vary in answer correction types. They remained to be significant predictors even after adjusting for respondent's characteristics and location, with interviewer clustering accounted as a fixed effect. At the same time, the correction patterns based on the number of corrections per question and answer correction types were very similar between the two reproductive modules. The latter two were also not found to be significantly associated with the probability of making answer corrections and the number of answer corrections.

Even though the median number of corrected questions per interview and corrections per one question was relatively low (2 and 1), the maximum numbers of corrected questions and corrections per question were high (110 and 28, respectively). Not only does this add to the duration of section completion (as shown in our results), and ultimately to interview duration, it could also potentially shed light on questions that are poorly understood or misunderstood by either respondent or interviewer. For example, in our survey, the highest proportion of corrections was among the numerical computational (9.7\%) and TOP-related (8.0\%) questions. At the same time, even higher proportions of corrections were observed for multi-select questions (44.8\%) and free-text (16.2\%) questions. They also had the strongest association with the probability of making answer corrections. However, these results should be treated with caution as these questions constitute less than $0.1 \%$ among all questions asked.

Moreover, given the design and related data entry process (i.e., sequential data entry for a combination of multiple answer options and noting down and correcting 
Table 3 Classification of answer correction type and possible explanations

\begin{tabular}{|c|c|c|c|c|c|}
\hline $\begin{array}{l}\text { Answer correction pattern } \\
\text { (from first to final answer } \\
\text { entry) }\end{array}$ & $\begin{array}{l}\text { Multiple }(>1) \\
\text { corrections }\end{array}$ & $\begin{array}{l}\text { First and last } \\
\text { entry match }\end{array}$ & Correction type & Problematic & Possible explanation \\
\hline $\mathrm{A}^{\mathrm{a}}$ & NO & YES & No correction & NO & $\mathrm{n} / \mathrm{a}$ \\
\hline$A>A>A$ & YES & YES & Impulsive & NO & $\begin{array}{l}\text { Multiple tapping (e.g., due to tablet's } \\
\text { lag response) }\end{array}$ \\
\hline$A>B^{b}$ & NO & NO & Flat (simple) correction & NO & $\begin{array}{l}\text { Accidental mistake, correction, following } \\
\text { clarification or mistake }\end{array}$ \\
\hline$A>n^{c}>A$ & YES & YES & Zigzag correction & YES & $\begin{array}{l}\text { Corrections after multiple additional } \\
\text { clarifications and confirmation (e.g., } \\
\text { change in responses) and changed } \\
\text { back to the original answer }\end{array}$ \\
\hline$A>n>B$ & YES & NO & Flat zigzag correction & YES & Correction, following clarifications \\
\hline$A>\ldots$ & NO & NO & Missing after correction & YES & $\begin{array}{l}\text { Accidental mistake corrected after } \\
\text { clarification, request to skip or not } \\
\text { to record the answer }\end{array}$ \\
\hline $\begin{array}{l}A>B>\ldots \text { or } A>n>B>\ldots \text { or } \\
A>n>A>\ldots\end{array}$ & YES & NO & $\begin{array}{l}\text { Missing after flat (zigzag) } \\
\text { correction }\end{array}$ & YES & $\begin{array}{l}\text { Clarification and consequent refusal } \\
\text { to answer or request to skip or not } \\
\text { to record the answer }\end{array}$ \\
\hline & NO & YES & Missing/incomplete & YES & Unknown \\
\hline
\end{tabular}

${ }^{a}$ stands for the original (first entered) answer

${ }^{b}$ represents a different answer in content than the original answer $\mathrm{A}$

crepresents a number of answer corrections between first and last answer in sequence

the free text), distinguishing between answers, answer combinations, and corrections might not always be straightforward. Nonetheless, we suggest that these types of questions get additional attention during training sessions, with more time being allocated to explaining and practising asking these questions, with additional guidance and supervision provided during the fieldwork. One could also consider limiting even further these types of questions in household surveys.
We also developed and tested a new classification of answer correction types. We found this classification useful and suggest it for future studies. For example, we found that almost $90 \%$ of all corrections were simple mistake corrections (which is less worrisome) or impulsive and repetitive answers (most likely due to non-responsive screen); hence, they should not be considered problematic. This suggested that the reasons for the remaining multiple zigzag corrections (around 10\%) lie elsewhere. We speculate

Table 4 Answer correction types per question type, nature, and structure

\begin{tabular}{|c|c|c|c|c|c|c|c|c|c|}
\hline \multirow[t]{2}{*}{ Question characteristics } & \multirow[t]{2}{*}{$n$} & \multicolumn{2}{|c|}{ Flat (simple) } & \multicolumn{2}{|c|}{ Impulsive } & \multicolumn{2}{|c|}{ Zigzag correction } & \multicolumn{2}{|c|}{ Flat zigzag correction } \\
\hline & & $n$ & $\%$ & $n$ & $\%$ & $n$ & $\%$ & $n$ & $\%$ \\
\hline Overall & 250,608 & 212,057 & 84.6 & 11,438 & 4.6 & 13,288 & 5.3 & 13,825 & 5.5 \\
\hline \multicolumn{10}{|l|}{ Type } \\
\hline Single-select & 147,157 & 123,818 & 84.1 & 7613 & 5.2 & 10,124 & 6.9 & 5602 & 3.8 \\
\hline Multi-select & 86 & 47 & 54.7 & 4 & 4.7 & 6 & 7.0 & 29 & 33.7 \\
\hline Numerical computational & 101,607 & 86,850 & 85.5 & 3604 & 3.5 & 3080 & 3.0 & 8073 & 7.9 \\
\hline Date-related & 1691 & 1290 & 76.3 & 213 & 12.6 & 76 & 4.5 & 112 & 6.6 \\
\hline Free-text & 67 & 52 & 77.6 & 4 & 6.0 & 2 & 3.0 & 9 & 13.4 \\
\hline \multicolumn{10}{|l|}{ Nature } \\
\hline Regular & 221,447 & 188,670 & 85.2 & 9917 & 4.5 & 10,774 & 4.9 & 12,086 & 5.5 \\
\hline Death-related & 22,628 & 17,871 & 78.9 & 1390 & 6.1 & 1905 & 8.4 & 1462 & 6.5 \\
\hline TOP-related & 6533 & 5516 & 84.4 & 131 & 2.0 & 609 & 9.3 & 277 & 4.2 \\
\hline \multicolumn{10}{|l|}{ Structure } \\
\hline Built-in error notification & 99,532 & 85,244 & 85.6 & 3492 & 3.5 & 2979 & 3.0 & 7817 & 7.9 \\
\hline No built-in error notification & 151,076 & 126,813 & 83.9 & 7946 & 5.3 & 10,309 & 6.8 & 6008 & 4.0 \\
\hline
\end{tabular}


that the remaining corrections were made following additional clarifications or confirmations of previous or later answers (Table 1). However, to verify our assumptions and identify other factors that affect correction probability and frequency (e.g., the exact wording or any other contextual factors) and even further unpack reasons behind answer corrections, field observations and qualitative interviews with both respondents and interviewers will be necessary. This once again underlines the importance of adequate timing dedicated to the data collection training, extensive field questionnaire testing, and effective supervision and guidance.

We believe that our proposed classification of answer correction types accounts for several dimension of the answer correction process. However, we would like to invite other researchers to evaluate our classification, improve and optimise it further, and test its usefulness and applicability in other types of surveys and research settings. Using our survey data, we identified only five answer correction types (including no corrections), lacking missing or incomplete answers after correction. This is primarily due to a lack of 'missing' (or empty in content) timestamped entries in our paradata dataset (which by default is not possible) and our decision not to treat 'Do not know' answers as 'missing' entries. However, we also much acknowledge effective training and comprehensive training manuals, diligent work of our data collectors, and their dedication to prompt interviewees and complete all relevant fields, which we believe aspired minimisation of any missing data. About a third of all questions in our questionnaire had inbuilt error notification, prompts, and warnings. Our results suggest that such notifications are effective since the proportion of corrected questions was 3.3\% higher for questions with notifications compared to those without them, and having notifications was significantly associated with the probability of making corrections but negatively associated with the number of such corrections. Additionally, Survey Solutions application had a built-in colour coding indicating survey section completion (red for incomplete and unanswered questions and green for complete), which prompted data collectors to answer all questions. For example, during some training sessions at several data collection sites, interviewers were insisting on learning how to achieve completeness 'having all sections colour-coded as green' in all survey sections and were 'somewhat unhappy' to finish the exercise with one or more sections remaining incomplete (or red).

\section{Strength and limitations}

Given our focus on corrections during interviewing, in our analysis, we excluded implausible and impractical values (over $180 \mathrm{~min}$ for section completion duration) but allowed for multiple corrections during the module completion and switching between questions and questionnaire sections. We assumed that such restrictions could provide meaningful and practical insights into face-to-face data collection process, even if it would exclude and not account for long breaks in the interviews (stopped and resumed several days/weeks/ months later) or other errors in timestamps (e.g., resulting from a change of a tablet's calendar set up from local form to the Gregorian calendar during data collection). However, we recognise that this decision could be considered as one of the limitations of the study, as it potentially did not capture corrections based on office data quality and error checks (following which questionnaires were returned and/or reassigned back to the interviewer for correction in-field). Moreover, in our analyses, we specifically focused only on a subset of paradata that related to answers and corrections. We did not utilise the data with timestamped events that related to process-related activities (e.g., interviewer or supervisor comments; enabling and disabling questions; declaring answers as valid or invalid based on the passing or failing of programmed validation rules; switching between the questionnaire's translations; recalculating system variable values based on manual correction), which could be considered another limitation of the study. As paradata were not readily available for export at the beginning of our data collection (due to software limitations), we did not evaluate individual and team productivity (e.g., average hours per contact attempt, contact attempts without success, number of interviews per workday), or estimate the response likelihood and perform measurement error evaluation [6]. However, we relied on experience from our local data collection teams and invested additional time into training and field testing.

\section{Research gaps for improving measurements of $\mathrm{MNCH}$ indicators in household surveys}

Given a lack of other studies that have used paradata in $\mathrm{MNCH}$ field, we cannot compare our findings directly to other studies, and we would like to stimulate the wider use of survey paradata to advance survey design and implementation for collecting information on pregnancies and births and for other purposes.

Paradata provide a wealth of information and could augment surveys, particularly overseeing the data collection process. Not surprisingly, it has already found use in other health and medical areas. For example, similar to our study, paradata were previously used in telemedicine research to estimate time spent to complete a questionnaire [20] and to examine completion and impact of push notifications on data completion in behaviour risk assessment [21]. Other applications in 
health include examining the role of paradata in nonresponse adjustment process [22], underreporting errors and finding suggestions for methodological improvement for future surveys [23] and examining response time at the level of individual questions [24]. Other studies examined practical use of paradata, for example, as an interactive web-based data visualisation tool, providing survey staff with the information to monitor data collection daily [25]. Using paradata (along with metadata and embedded data) can also improve response rates, identify bias, and give a possible explanation for apparent outlier responses, providing an efficient method of conducting web-based Delphi surveys [26]. Overall, using paradata in health research suggests that paradata could be valuable in quantifying recruitment efforts and aid the development and evaluation of new recruitment strategies [27].

Future analyses could investigate the relationship between correction rates and correction type and being supervised by a supervisor or other colleagues, which potentially could prompt additional corrections under peer pressure. Other potential uses of paradata in $\mathrm{MNCH}$ research could include effort indicators, tracking individual and team productivity, estimating contact attempts without success, and response propensity. Outcome indicators and case status indicators can also include non-interviews by type and refusal patterns by respondent characteristics. Paradata in $\mathrm{MNCH}$ research can also be used to generate a dashboard/monitoring system or a validation system for collected data against external sources of information, hence, automatically flagging incorrect entries in the interviews.

\section{Conclusion}

Accurate estimation of coverage indicators from household surveys is vital but contingent on data quality; hence, a better understanding of how to improve the questionnaire design and survey implementation is crucial. Paradata have the potential to enhance survey design and implementation for collecting information on pregnancies and births, leading to improved metrics of measurement in maternal and newborn health research. They can help to identify questions and sections with multiple corrections and shed light on typically hidden challenges in the survey's content, process, and administration. Overall, our experience suggests that given the size of paradata and their complex structure, analysis is not always straightforward, and consideration should be given to the additional data management and programming skills required. Nonetheless, paradata provide a wealth of data, can improve the process of data collection using live survey monitoring, and can add value in improving survey data quality as well as efficiency.

\section{Supplementary Information}

The online version contains supplementary material available at https://doi.org/10.1186/s12963-020-00241-0.

Additional file 1. Detailed overview of questions in Section 2 in $\mathrm{FPH}$ and $\mathrm{FBH}+$.

Additional file 2. Example of survey paradata structure.

Additional file 3. STROBE guidelines checklist.

Additional file 4. Types of questions in Section 2 in $\mathrm{FPH}$ and $\mathrm{FBH}+$.

Additional file 5. Detailed overview of questions correction by question type, content and structure and reproductive module.

Additional file $\mathbf{6}$. Correction patterns, 23 groups by the number of corrections per question.

Additional file 7. Ethical approval of local Institutional Review Boards.

\section{Abbreviations}

CAPI: Computer-assisted personal interviewing; CAWI: Computer-assisted web interviewing; DHS: Demographic and Health Surveys; EN-INDEPTH: Every Newborn International Network for the Demographic Evaluation of Populations and Their Health; FBH+: Full birth history (+ denotes additional questions on pregnancy losses); FPH: Full pregnancy history; HDSS: Health and Demographic Surveillance System; LMIC: Low- and middle-income countries; MICS: Multiple Indicator Cluster Survey; MNCH: Maternal, newborn, and child health; TOP: Termination of pregnancy

\section{Acknowledgements}

This supplement is dedicated to the memory of Professor Peter Byass, who was the Senior External Editor of the supplement. Peter died suddenly in August 2020 and will be greatly missed by the EN-INDEPTH study team and the entire global health community.

We thank the 118 interviewers and many Health and Demographic Surveillance System staff participating in this study for their hard work and dedication to this project. Many thanks to Samuelina Arthur, Claudia DaSilva, Olivia Nakisita, Maria Cesay, and the relevant site staff for their administrative support.

We acknowledge the core funders for all sites/institutions.

We express appreciation to the EN-INDEPTH expert advisory group: Fred Arnold, Peter Byass, Trevor Croft, Kobus Herbst, Sunita Kishor, Florina Serbanescu, Stephane Helleringer, Turgay Unalan, Shane Khan, Attila Hancioglu. Additionally, we would like to thank members of the World Bank Survey Solutions technical support team for their guidance and support during survey implementation.

Finally, and most importantly, we thank the women participating in the ENINDEPTH study and their families, without whom this work would not have been possible.

The Every Newborn-INDEPTH Study Collaborative Group:

Senior External Supplement Editors: Peter Byass; Stephen M Tollman; Hagos Godefay

Technical Supplement Editors: Joy E. Lawn; Peter Waiswa; Hannah Blencowe

Managing Supplement Editors: Judith Yargawa; Joseph Akuze (data and statistics)

Other EN-INDEPTH Collaborative Group Members:

By team: PI followed by other members in alphabetical order

Bandim: Ane B Fisker (PI); Justiniano SD Martins; Amabelia Rodrigues; Sanne M Thysen

Dabat: Gashaw Andargie Biks (PI); Solomon Mokonnen Abebe; Tadesse Awoke Ayele; Telake Azale Bisetegn; Tadess Guadu Delele; Kassahun Alemu Gelaye; Bisrat Misganaw Geremew; Lemma Derseh Gezie; Tesfahun Melese; Mezgebu Yitayal Mengistu; Adane Kebede Tesega; Temesgen Azemeraw Yitayew

IgangaMayuge: Simon Kasasa (PI); Edward Galiwango; Collins Gyezaho; Judith Kaija; Dan Kajungu; Tryphena Nareeba; Davis Natukwatsa; Valerie Tusubira

Kintampo: Yeetey AK Enuameh (PI); Kwaku P Asante; Francis Dzabeng; Seeba Amenga-Etego; Alexander A Manu; Grace Manu; Obed Ernest Nettey; Sam K Newton; Seth Owusu-Agyei; Charlotte Tawiah; Charles Zandoh

Matlab: Nurul Alam (PI); Nafisa Delwar; M Moinuddin Haider; Md. Ali Imam; Kaiser Mahmud 
LSHTM/ Makerere School of Public Health: Angela Baschieri; Simon Cousens; Vladimir Sergeevich Gordeev; Victoria Ponce Hardy; Doris Kwesiga; Kazuyo Machiyama

\section{About this supplement}

This article has been published as part of Population Health Metrics Volume 19 Supplement 1, 2021: Every Newborn-INDEPTH study: Improving the measurement of pregnancy outcomes in population-based surveys. The full contents of the supplement are available online at https://pophealthmetrics. biomedcentral.com/articles/supplements/volume-19-supplement-1.

\section{Authors' contributions}

JEL conceptualised the EN-INDEPTH study. All EN-INDEPTH collaborative group and site teams contributed to the design of the study protocol and undertook data collection. VSG, together with HB and JEL, developed the detailed research questions and overall analysis plan for this paper. These were refined with inputs from the wider EN-INDEPTH study collaborative group including $A B$ at a multi-country workshop in Entebbe Uganda in February 2019. SMT, FD, MMH, TAY, SMA, DN, CG, and SIA implemented the study at the sites. MW and ML were consulted specifically on the Survey Solutions software. JA prepared the survey data files. VSG and MS undertook quantitative data analysis. The manuscript was drafted by VSG, with inputs from HB and JEL. All authors reviewed and agreed on the final version of the manuscript.

\section{Funding}

The EN-INDEPTH study (including publication costs) was funded by the Children's Investment Fund Foundation (CIFF) by means of a grant to LSHTM (PI Joy E. Lawn) and a sub-award to the INDEPTH MNCH working group with technical leadership by Makerere School of Public Health (PI Peter Waiswa).

\section{Availability of data and materials}

Data sharing and transfer agreements were jointly developed and signed by all collaborating partners. The datasets generated during the current study are deposited online at https://doi.org/10.17037/DATA.00001556 with data access subject to approval by collaborating parties.

\section{Ethics approval and consent to participate}

The EN-INDEPTH study was granted ethical approval by the Institutional Review Boards in all operating countries, as well as, from the Institutional Ethical Review Committee of the London School of Hygiene \& Tropical Medicine (Additional file 7). Respondents of every successful interview gave written consent/ascent after being informed of the objective and data use, the procedure of the interview, risks and benefits of participating in the study, right to withdraw from interview anytime point of time, and not responding to questions where they feel discomfort. The study ensures the respondent's privacy at data collection and confidentiality at data use.

\section{Consent for publication}

Not applicable.

\section{Competing interests}

The authors declare no competing interests.

\section{Author details}

${ }^{1}$ Institute of Population Health Sciences, Queen Mary University of London, London, UK. ${ }^{2}$ Maternal, Adolescent, Reproductive \& Child Health (MARCH) Centre, London School of Hygiene \& Tropical Medicine, London, UK. ${ }^{3}$ Department of Health Policy, Planning and Management, Makerere University School of Public Health, Kampala, Uganda. ${ }^{4}$ Centre of Excellence for Maternal Newborn and Child Health Research, Makerere University, Kampala, Uganda. ${ }^{5}$ Bandim Health Project, Bissau, Guinea-Bissau. ${ }^{6}$ Research Centre for Vitamins and Vaccines, Statens Serum Institut, Copenhagen, Denmark. ${ }^{7}$ Department of Clinical Research Open Patient data Explorative Network (OPEN), University of Southern Denmark, Odense, Denmark. ${ }^{8}$ Kintampo Health Research Centre, Kintampo, Ghana. ${ }^{9}$ Health Systems and Population Studies Division, icddr,b, Dhaka, Bangladesh. ${ }^{10}$ Department of Medical Statistics, London School of Hygiene \& Tropical Medicine, London, UK. ${ }^{11}$ The World Bank, Washington DC, USA. ${ }^{12}$ Dabat Research Centre Health and Demographic Surveillance System, Dabat, Ethiopia. ${ }^{13}$ IgangaMayuge Health and Demographic Surveillance System, Makerere University Centre for Health and Population Research, Makerere, Uganda.
Published: 8 February 2021

\section{References}

1. Commission on Information Accountability for Women's and Children's Health. Keeping promises, measuring results: commission on information and accountability for women's and children's health. Geneva: Commission on information and accountability for Women's and Children's Health, World Health Organization; 2011.

2. Countdown to 2015 \& Health Metrics Network. Monitoring maternal, newborn and child health: understanding key progress indicators. Geneva: World Health Organization; 2011.

3. Hansen SE, Benson G, Bowers A, Pennell B-E, Lin Y-cJ, Duffey B, Hu M, Hibben KC. Survey quality. In: Guidelines for best practice in cross-cultural surveys. Ann Arbor: Survey Research Center, Institute for Social Research, University of Michigan; 2016.

4. Hancioglu A, Arnold F. Measuring coverage in MNCH: tracking progress in health for women and children using DHS and MICS household surveys. PLoS Med. 2013;10:e1001391.

5. Leisher C. A comparison of tablet-based and paper-based survey data collection in conservation projects. Soc Sci. 2014;3:264-71.

6. Kreuter F, editor. Improving surveys with paradata: analytic uses of process information. Hoboken: Wiley; 2013.

7. Nicolaas G: Survey paradata: a review. 2011. http://eprints.ncrm.ac.uk/171 9/1/Nicolaas_review_paper_jan11.pdf. [Accessed June 2020].

8. Choumert-Nkolo J, Cust H, Taylor C. Using paradata to collect better survey data: evidence from a household survey in Tanzania. Rev Dev Econ. 2019;23:598-618.

9. Couper MP, Kreuter F. Using paradata to explore item level response times in surveys. J R Stat Soc Ser A Stat Soc. 2013;176:271-86.

10. Baschieri A, Gordeev VS, Akuze J, Kwesiga D, Blencowe H, Cousens S, Waiswa P, Fisker AB, Thysen SM, Rodrigues A, et al. "Every Newborn-INDEPTH" (EN-INDEPTH) study protocol for a randomised comparison of household survey modules for measuring stillbirths and neonatal deaths in five Health and Demographic Surveillance sites. J Glob Health. 2019; 9:010901.

11. Akuze J, Blencowe H, Waiswa P, Baschieri A, Gordeev VS, Kwesiga D, Fisker AB, Thysen SM, Rodrigues A, Biks GA, et al. Randomised comparison of two household survey modules for measuring stillbirths and neonatal deaths in five countries: the Every Newborn-INDEPTH study. Lancet Glob Health. 2020;8:e555-66.

12. The World Bank. Survey solutions CAPI/CAWI platform: release 5.26. Washington DC: The World Bank; 2018.

13. Thysen SM, Tawiah C, Blencowe H, Manu G, Akuze J, Haider MM, Alam N, Azemeraw T, Baschieri A, Biks GA, et al. Electronic data collection in a multi-site population-based survey: EN-INDEPTH study. BMC Population Health Metrics. 2021:19(Supplement 1).

14. R Core Team. R: a language and environment for statistical computing. Vienna: R Foundation for Statistical Computing; 2019.

15. StataCorp. Stata Statistical Software: release 16. College Station: StataCorp LLC; 2019.

16. Yan T, Olson K. Analysing paradata to investigate measurement error. In: Kreuter F, editor. Improving surveys with paradata: analytic uses of process information. Hoboken: Wiley; 2013. p. 73-95.

17. Brzinsky-Fay C, Kohler U, Luniak M. Sequence analysis with Stata. Stata J. 2006;6:435-60.

18. von Elm E, Altman DG, Egger M, Pocock SJ, Gøtzsche PC, Vandenbroucke JP; STROBE Initiative. Strengthening the Reporting of Observational Studies in Epidemiology (STROBE) statement: guidelines for reporting observational studies. BMJ. 2007;335(7624):806-8. https://doi.org/10.1136/bmj.39335. 541782.AD. PMID: 17947786; PMCID: PMC2034723.

19. Elliott RN. The wave principle. Los Angeles: Alanpuri Trading; 1938.

20. Romano MF, Sardella MV, Alboni F. Web health monitoring survey: a new approach to enhance the effectiveness of telemedicine systems. JMIR Res Protoc. 2016;5:e101.

21. Oakley-Girvan I, Lavista JM, Miller Y, Davis S, Acle C, Hancock J, Nelson LM. Evaluation of a mobile device survey system for behavioral risk factors (SHAPE): app development and usability study. JMIR Form Res. 2019;3:e10246.

22. Substance Abuse and Mental Health Services Administration (US). CBHSQ methodology report. In: Incorporating level of effort paradata in the NSDUH nonresponse adjustment process. Rockville: Substance Abuse and Mental Health Services Administration (US); 2013

23. Hu M, Gremel GW, Kirlin JA, West BT. Nonresponse and underreporting errors increase over the data collection week based on paradata from the National Household Food Acquisition and Purchase Survey. J Nutr. 2017;147:964-75. 
24. Craig BM, Runge SK, Rand-Hendriksen K, Ramos-Goni JM, Oppe M. Learning and satisficing: an analysis of sequence effects in health valuation. Value Health. 2015;18:217-23.

25. Murphy JJ, Duprey MA, Chew RF, Biemer PP, Harris KM, Halpern CT. RTI press occasional papers. In: Interactive visualization to facilitate monitoring longitudinal survey data and paradata. Research Triangle Park: Research Triangle Institute (RTI) Press; 2019.

26. Helms C, Gardner A, McInnes E. The use of advanced web-based survey design in Delphi research. J Adv Nurs. 2017;73:3168-77.

27. Langeheine M, Pohlabeln H, Ahrens W, Rach S. Consequences of an extended recruitment on participation in the follow-up of a child study: results from the German IDEFICS cohort. Paediatr Perinat Epidemiol. 2017;31:76-86.

\section{Publisher's Note}

Springer Nature remains neutral with regard to jurisdictional claims in published maps and institutional affiliations.

Ready to submit your research? Choose BMC and benefit from:

- fast, convenient online submission

- thorough peer review by experienced researchers in your field

- rapid publication on acceptance

- support for research data, including large and complex data types

- gold Open Access which fosters wider collaboration and increased citations

- maximum visibility for your research: over $100 \mathrm{M}$ website views per year

At $\mathrm{BMC}$, research is always in progress.

Learn more biomedcentral.com/submissions 